\title{
Effects of Hippocampal State-Contingent Trial Presentation on Hippocampus-Dependent Nonspatial Classical Conditioning and Extinction
}

\author{
Miriam S. Nokia and Jan Wikgren \\ Department of Psychology and Jyväskylä Centre for Interdisciplinary Brain Research, University of Jyväskylä, Jyväskylä 40014, Finland
}

\begin{abstract}
Hippocampal local field potentials are characterized by two mutually exclusive states: one characterized by regular $\theta$ oscillations $(\sim 4-8$ $\mathrm{Hz})$ and the other by irregular sharp-wave ripples. Presenting stimuli during dominant $\theta$ oscillations leads to expedited learning, suggesting that $\theta$ indexes a state in which encoding is most effective. However, ripple-contingent training also expedites learning, suggesting that any discrete brain state, much like the external context, can affect learning. We trained adult rabbits in trace eyeblink conditioning, a hippocampus-dependent nonspatial task, followed by extinction. Trials were delivered either in the presence or absence of $\theta$ or regardless of hippocampal state. Conditioning in the absence of $\theta$ led to more animals learning, although learning was slower compared with a yoked control group. Contrary to expectations, conditioning in the presence of $\theta$ did not affect learning. However, extinction was expedited both when it was conducted contingent on $\theta$ and when it was conducted in a state contrary to that used to trigger trials during conditioning. Strong phase-locking of hippocampal $\theta$-band responses to the conditioned stimulus early on during conditioning predicted good learning. No such connection was observed during extinction. Our results suggest that any consistent hippocampal oscillatory state can potentially be used to regulate learning. However, the effects depend on the specific state and task at hand. Finally, much like the external environment, the ongoing neural state appears to act as a context for learning and memory retrieval.
\end{abstract}

Key words: classical conditioning; extinction; hippocampus; $\theta$

\section{Introduction}

Hippocampal local field potentials (LFPs) are characterized by two mutually exclusive states: highly rhythmic $\theta(\sim 4-8 \mathrm{~Hz})$ (Green and Arduini, 1954; see also Buzsáki and Moser, 2013) and irregular sharp-wave activity, during which ripples $(\sim 200 \mathrm{~Hz})$ (Chrobak and Buzsáki, 1996) are present. Spontaneous $\theta$ oscillations in an awake animal are thought to reflect a state in which labile memory traces of events that are taking place are formed (Buzsáki, 1989), the read-state. Consistently, a high relative power of hippocampal $\theta$ activity before training predicts good learning (Berry and Thompson, 1978; Nokia et al., 2012a), and presenting trials contingent on periods of high $\theta$ facilitates hippocampus-dependent trace eyeblink classical conditioning (Griffin et al., 2004). Interestingly, presenting conditioning trials contingent with ripples (in the absence of $\theta$ ) also expedites learning (Nokia et al., 2010). Thus, it is unclear whether presenting trials contingent on any consistent neural state would facilitate

Received Nov. 19, 2013; revised March 20, 2014; accepted March 24, 2014.

Author contributions: M.S.N. and J.W. designed research; M.S.N. and J.W. performed research; M.S.N. analyzed data; M.S.N. and J.W. wrote the paper.

This work was supported by Academy of Finland Grant 137783 to M.S.N. We thank Lauri Viljanto and Jarno Mikkonen for technical help; Kaisu Myllys and Saara Johansson for help with data acquisition; and Michael Freeman for editorial help.

The authors declare no competing financial interests.

Correspondence should be addressed to Dr. Miriam S. Nokia, Jyväskylä Centre for Interdisciplinary Brain Research, University of Jyväskylä, P.0. Box 35, 40014 Jyväskylä, Finland. E-mail: miriam.nokia@jyu.fi.

DOI:10.1523/JNEUROSCI.4859-13.2014

Copyright $\odot 2014$ the authors $\quad 0270-6474 / 14 / 346003-08 \$ 15.00 / 0$ learning. In addition, good learning is predicted not only by the spontaneously ongoing oscillatory state, but also strong and well phase-locked hippocampal $\theta$-band responses to the conditioned stimulus (CS) (Nokia et al., 2009, 2010; Nokia and Wikgren, 2010). Whether learning is predominantly dependent on the pretrial hippocampal oscillatory state, which then determines subsequent responses to the CS, or whether the responses themselves determine learning, is not clear.

Most studies on the role of hippocampal oscillatory activity in learning have overlooked learning modifications to already acquired associations. Discriminatory reversal learning does not seem to correlate with hippocampal $\theta$-band responses to conditioning stimuli (Nokia and Wikgren, 2010), but presenting extinction trials contingent on hippocampal ripples hinders extinction (Nokia et al., 2010). To our knowledge, whether hippocampal $\theta$ is connected to simple extinction has not been studied. It is also not known whether an internal state characterized by consistent hippocampal oscillations could, much like the external environment, work as a context for learning (Godden and Baddeley, 1975).

To address the issues raised above, we trained adult female New Zealand White rabbits in trace eyeblink conditioning using a $500 \mathrm{~ms}$ trace interval. Training trials were timed to occur when endogenous hippocampal $\theta$ was either prominent $\left(\mathrm{T}^{+}\right)$or absent $\left(\mathrm{T}^{-}\right)$. Animals in the respective yoked control (YC) groups received training trials regardless of their brain activity. For animals that acquired a robust conditioned response, conditioning was 
followed by extinction. For this, half the animals in the $\mathrm{T}^{+}$group were switched to the $\mathrm{T}^{-}$group and vice versa. We expected better phase-locked hippocampal $\theta$-band responses to the CS and better and faster conditioning in both the $\mathrm{T}^{+}$and the $\mathrm{T}^{-}$groups compared with animals trained regardless of their neural state. In addition, we assumed a predictive correlation between well phase-locked $\theta$-band responses to the CS and good learning. Finally, we hypothesized that extinction would be facilitated in both the $\mathrm{T}^{+}$group and in animals that were trained contingent on one hippocampal state and extinguished in a different state $\left(\mathrm{T}^{+} / \mathrm{T}^{-}\right.$or $\left.\mathrm{T}^{-} / \mathrm{T}^{+}\right)$.

\section{Materials and Methods}

\section{Subjects}

The subjects were 36 adult female New Zealand White rabbits (Lidköpings kaninfarm), $\sim 4$ months of age and weighing $\sim 2.7 \mathrm{~kg}$ at the time of surgery. The rabbits were housed in individual cages on the premises of the animal research unit of the University of Jyväskylä. Food and water were freely available, and room temperature and humidity were controlled. The rabbits were maintained on a $12 / 12 \mathrm{~h}$ light/dark cycle, with lights on at 8:00 A.M. All procedures were conducted during the light portion of the cycle. All the experimental procedures were implemented in accordance with directive 2010/63/EU of the European Parliament and of the Council on the care and use of animals for research purposes.

\section{Surgery}

Under general anesthesia, four monopolar recording electrodes made of Teflon-insulated stainless steel wire (bare diameter $125 \mu \mathrm{m}$ ) mounted inside 27-gauge hypodermic stainless steel tubing were chronically implanted into the left dorsal hippocampus $5 \mathrm{~mm}$ posterior, $4-7 \mathrm{~mm}$ lateral, and $6.5-7.4 \mathrm{~mm}$ below the bregma, aiming at the hippocampal fissure and dentate gyrus (for details on surgery, see Nokia et al., 2012b). Four stainless-steel anchoring screws were attached to the skull. The screws were connected in pairs and served as ground and reference for the LFPs. The electrodes were attached to a pin connector and the whole construction cemented in place with dental acrylic. At least 1 week was allowed for postsurgical recovery.

\section{Conditioning procedure}

Before the experiments, the rabbits were placed $(\sim 20 \mathrm{~min})$ in a Plexiglas restraining box located in a ventilated, electrically insulated, and soundattenuated conditioning chamber to familiarize them with the experimental situation, and to ensure the functioning of the implanted electrodes. Thereafter, experimental sessions were conducted once per day on consecutive days, excluding weekends.

The CS was a $4 \mathrm{kHz}, 85 \mathrm{~dB}, 200 \mathrm{~ms}$ tone, and the unconditioned stimulus (US) was a $100 \mathrm{~ms}$ corneal air puff ( 0.35 bar source pressure, 64 $\mathrm{dB}$ sound pressure level) delivered through a nozzle (inner diameter 2 $\mathrm{mm}$ ) placed $\sim 1 \mathrm{~cm}$ away from the eye. A fan located inside the conditioning chamber created a steady background noise $(65 \mathrm{~dB})$. LabView (National Instruments) and E-Prime software (Psychology Software Tools) were used to control the presentation of stimuli.

First, an unpaired session was conducted to obtain baseline measures. Both the CS and the US were presented alone 60 times, in random order and regardless of the hippocampal state, with an intertrial interval (ITI) of 15-25 s. Next, the rabbits were pseudo-randomly assigned to three groups: rabbits in the $\mathrm{T}^{+}$group $(n=11)$ received training trials contingent on high hippocampal $\theta$ activity; rabbits in the $\mathrm{T}^{-}$group $(n=10)$ received trials when hippocampal $\theta$ activity was low; and rabbits in the YC group $(n=15)$ were trained paired with either a $\mathrm{T}^{+}(n=8)$ or a $\mathrm{T}^{-}$ $(n=7)$ rabbit and thus received trials regardless of their neural state. All rabbits were trained in trace eyeblink conditioning. Each session consisted of 60 paired trials where a $500 \mathrm{~ms}$ silent trace period separated the $\mathrm{CS}$ from the following US. The minimum ITI was set to $15 \mathrm{~s}$ and varied according to the hippocampal oscillatory state of the $\mathrm{T}^{+} / \mathrm{T}^{-}$animal. Conditioning was ceased when the animal reached the predetermined learning criterion of $\geq 60 \%$ conditioned responses (CRs) in at least two out of three consecutive training sessions. A minimum of 10 and a max- imum of 16 conditioning sessions were conducted. If an animal reached the criterion of $\geq 60 \%$ CRs in two out of three sessions within the minimum number of 10 sessions but performance was lower than $60 \%$ in session 10 , conditioning was continued until the animal again attained the $60 \%$ criterion.

All animals that had reached the $\geq 60 \%$ criterion during at least one conditioning session were subjected to 10 sessions of extinction training identical to the conditioning, except that the US was now omitted. For extinction, the rabbits in the $\mathrm{T}^{-}$and $\mathrm{T}^{+}$groups were reassigned so that half the animals remained in the same group and half switched from $\mathrm{T}^{+}$ to $\mathrm{T}^{-}$or vice versa. The animals in the YC group remained in that group also for extinction.

Triggering trials based on hippocampal $\theta$ ratio. The LFP signal from one hippocampal electrode was monitored online in consecutive, nonoverlapping, $1 \mathrm{~s}$ sweeps. From each sweep, the FFT was calculated with a resolution of $1 \mathrm{~Hz}$. The $\theta$ ratio was calculated as the ratio between the power of the signal at $3.5-8.5 \mathrm{~Hz}$ divided by the power of the signal at $1-8.5 \mathrm{~Hz}(\theta /[\delta+\theta])$. For animals in the $\mathrm{T}^{+}$group, trials were presented when the $\theta$ ratio was $>85 \%$. For animals in the $\mathrm{T}^{-}$group, trials were presented when the $\theta$ ratio was $<35 \%$. Occasionally, epochs of $\theta /$ no $-\theta$ fell in the middle of two $1 \mathrm{~s}$ sweeps sampled by Labview; thus, it was not necessarily always the first epoch of $\theta /$ no- $\theta$ (after the $15 \mathrm{~s}$ minimum ITI) that triggered a trial. If needed, the threshold for triggering was adjusted individually to an even more stringent value (e.g., $90 \%$ instead of $85 \%$ for an animal in the $\mathrm{T}^{+}$group), so that the state in which an animal was trained was one of an extremely high $\left(\mathrm{T}^{+}\right)$or low $\left(\mathrm{T}^{-}\right) \theta$ ratio in relation to that animal's general $\theta$ level.

\section{Recordings and data analyses}

Bipolar EMG was recorded using stainless steel wire hooks placed around the upper and lower eyelids for the duration of the training session. To acquire neural measures, a low-noise preamplifier (MPA8I, MultiChannel Systems) was directly attached to the electrode coupler anchored with dental acrylic to the rabbit's head. A flexible, insulated cable was used to connect the animal to the amplifier (Axon Cyberamp 380, Molecular Devices). All signals were recorded with AxoScope (Molecular Devices) software and digitized (Digidata 1322A, Molecular Devices) using a 2.02 $\mathrm{kHz}$ sampling rate. Before digitization, the LFPs were bandpass-filtered between 0.1 and $400 \mathrm{~Hz}$, and the EMG was filtered between 30 and 400 Hz. MATLAB (MathWorks) and SPSS (SPSS) were used for offline data analysis.

Baseline $\theta$. Data from the $20 \mathrm{~min}$ habituation session were analyzed offline to obtain baseline characteristics of hippocampal $\theta$-band activity. A sliding window of $1 \mathrm{~s}$ in $200 \mathrm{~ms}$ steps was used, and the FFT was calculated for each epoch. From the data, the duration and interevent interval were determined for the $\mathrm{T}^{+}(\theta$ ratio $>85 \%)$ and $\mathrm{T}^{-}(\theta$ ratio $<$ $35 \%$ ) epochs. To verify that our Labview routine used for triggering trials had worked as intended, we also calculated pretrial $\theta$ ratios offline for the first 10 conditioning sessions.

Eyeblinks. The EMG signal was high-pass filtered offline $(>100 \mathrm{~Hz})$ and Hilbert-transformed. An envelope curve following the peaks of the signal was calculated. Baseline EMG activity was defined for each animal and session as the mean of the peak EMG amplitude during a $500 \mathrm{~ms}$ pre-CS period (MEANpre). Also determined was the mean of the standard deviation (SD) of the EMG activity during the $500 \mathrm{~ms}$ pre-CS period (SDpre). Eyeblinks were defined as EMG activity exceeding a threshold of (MEANpre $+4 \times$ SDpre) for at least $10 \mathrm{~ms}$. Trials with eyeblinks during the $100 \mathrm{~ms}$ period immediately preceding CS onset were rejected. Eyeblinks during the latter half $(250 \mathrm{~ms}$ period $)$ of the $500 \mathrm{~ms}$ trace period were counted as conditioned responses. The learning criterion was considered to be met when the subject performed $\geq 60 \%$ CRs during at least one conditioning session. Learning rate was quantified as the number of conditioning sessions needed to reach the $60 \%$ criterion. We also measured learning rate as the number of trials needed to reach the traditional " 8 conditioned responses on 9 consecutive trials" criterion and the number of trials needed to perform the fifth CR.

Phase-locked hippocampal $\theta$-band $(4-8 \mathrm{~Hz}$ ) responses. To assess the temporal accuracy of $\theta$-band responses to the conditioning stimuli, a phase-locking value (PLV) (Palva et al., 2005) was calculated. The PLV is 
A

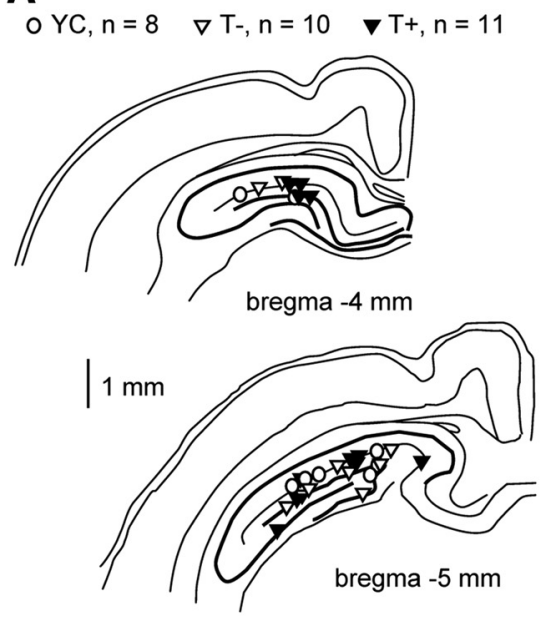

B

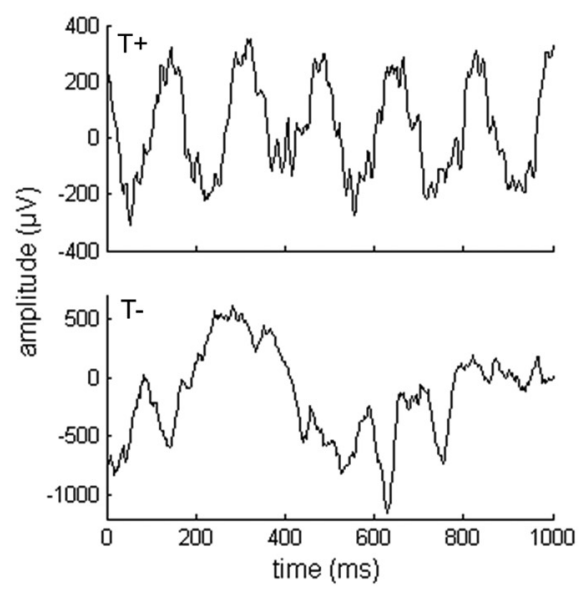

C

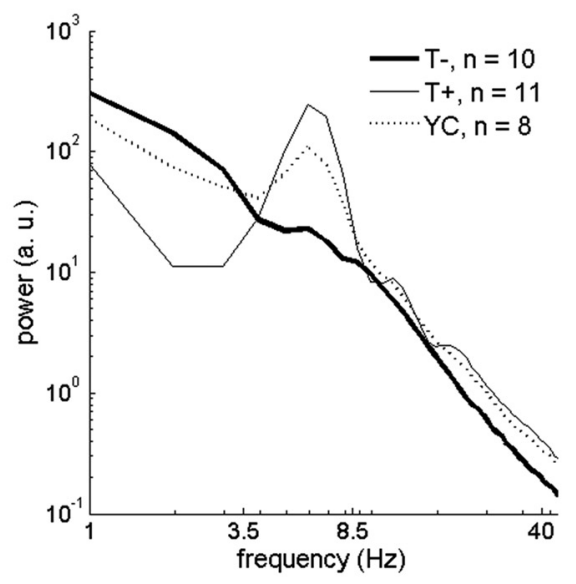

Figure 1. A, Electrodes had been correctly placed in the hippocampus in 29 of 36 animals. The 7 animals without hippocampal recordings were all in the $Y C$ group. $B$, Examples of LFPs with high $\left(\mathrm{T}^{+},>85 \%\right)$ and low $\left(\mathrm{T}^{-},<35 \%\right) \theta$ ratio. C, Power spectra corresponding to the pretrial periods during conditioning sessions $1-10$ illustrate the presence of $\theta$ in the $\mathrm{T}^{+}$group and the absence of $\theta$ in the $\mathrm{T}^{-}$group.

based on amplitude-normalized phase information and is thus resistant to changes or differences in signal amplitude. This allows comparable measures to be obtained from data recorded over time in multiple subjects. The hippocampal LFP data were first bandpass filtered between 4 and $8 \mathrm{~Hz}$. Then, a Hilbert transform was run on the signal to obtain the phase information, and the amplitude of the signal normalized to 1 by dividing each data point by its absolute value. Finally, the PLV was obtained by averaging over 60 trials (one session) and taking the absolute value of the mean. The PLV varies between 0 and 1 ( 0 indicating no phase locking and 1 indicating perfect phase locking). For statistical analyses, the mean of the PLV during the CS and subsequent trace period (700 ms) was derived and averaged over blocks of two sessions.

\section{Statistical analyses}

Repeated-measures ANOVA, with training blocks of two sessions (5) as a within-subjects factor and group as a between-subject factor, was used to analyze changes across training. Whenever a significant interaction of block and group was evident, separate repeated-measures ANOVAs were conducted for each group, using blocks as a within-subjects factor. Greenhouse-Geisser-corrected $p$ values were used when the sphericity assumption was violated according to Mauchly's test. One-way ANOVA was used in group comparisons when single variables were tested. Bonferroni corrected $p$ values were used for post hoc comparisons. The $\chi^{2}$ test was used to compare the proportions of animals that learned in each group. Pearson correlation coefficient was used to analyze the connection between hippocampal phase-locked $\theta$-band responses to the CS and learning. Finally, paired samples $t$ tests were used to analyze differences within subjects.

\section{Histology}

Rabbits were anesthetized with an intramuscular injection of ketaminexylazine mixture and then overdosed with an intravenous injection of pentobarbital (Mebunat vet, Orion-Yhtymä Oyj). Next, the brain was perfused with physiological saline followed by $9 \%$ formalin solution through the ascending aorta. The locations of the electrode tips were marked by passing a DC current $(200 \mu \mathrm{A}, 20 \mathrm{~s})$ through them. The brain was then removed and stored in formalin for several days. The brain was coronally sectioned with a vibratome into $60-\mu \mathrm{m}$-thick slices. The slices were attached to gelatinized slides, dried, and stained with Prussian blue and cresyl violet. The electrode-tip locations were determined with the help of a microscope.

\section{Results}

Histology and baseline hippocampal $\theta$ activity

One electrode per animal was selected for offline analyses based on the tip location and signal quality (Fig. 1). Twenty- nine of 36 animals had correctly placed electrodes in the hippocampus.

The average $\theta$ ratio (\%) as well as the duration, interval, proportion (of all studied epochs), and $\theta$ ratio of high and low $\theta$ epochs during the $20 \mathrm{~min}$ baseline recording were quantified and compared among the two experimental and two YC groups. Oneway ANOVAs revealed no differences between any of the groups in any of the baseline measures, $F_{(3,28)}=0.25-1.24$, not significant. The average $\theta$ ratio was $67 \%$ with an SEM of \pm 1.0 percentage unit. The animals were in a state characterized by a high hippocampal $\theta$ ratio $\left(\mathrm{T}^{+}, \theta\right.$ ratio $>85 \%$ ) for, on average, $29.71 \%$ ( \pm 1.86 percentage units) of the time. Epochs of high $\theta$ lasted, on average, for $1.57 \pm 0.03 \mathrm{~s}$ and occurred, on average, $2.70 \pm 0.08 \mathrm{~s}$ apart (onset-to-onset interval). The animals were in a state characterized by a low hippocampal $\theta$ ratio $\left(\mathrm{T}^{-}, \theta\right.$ ratio $\left.<35 \%\right)$ for, on average, $10.18 \%$ ( \pm 0.63 percentage units) of the time. Epochs of low $\theta$ lasted, on average, $1.25 \pm 0.01 \mathrm{~s}$ and occurred, on average, $4.95 \pm 0.37 \mathrm{~s}$ apart. Paired samples $t$ tests confirmed that $\mathrm{T}^{+}$ epochs were more frequent (onset-to-onset interval: $t_{(28)}=5.35$, $p<0.001$ ) and lasted longer (duration: $t_{(28)}=8.13, p<0.001$ ) than $\mathrm{T}^{-}$epochs.

To verify that our Labview routine had worked as intended, we calculated pretrial $\theta$ ratios offline for the first 10 conditioning sessions. The average $\theta$ ratio was $87 \% \pm 0.2$ percentage units in the $\mathrm{T}^{+}$group $(n=11)$ and $62 \% \pm 4.0$ percentage units in the respective YC group $(n=3)$. The average $\theta$ ratio was $22 \% \pm 1.5$ percentage units in the $\mathrm{T}^{-}$group $(n=11)$ and $57 \% \pm 1.7$ percentage units in the respective YC group $(n=5)$. Examples of episodes with a high and low hippocampal $\theta$ ratio that would trigger training trials in the two experimental groups are depicted in Figure $1 B$. The power spectra for the $1 \mathrm{~s}$ pretrial periods in the $\mathrm{T}^{-}$and $\mathrm{T}^{+}$groups are presented in Figure $1 C$.

\section{ITI variation across training and between groups}

During conditioning (all sessions, maximum 16), the mean $\left( \pm\right.$ SEM) ITI was $34.4 \pm 1.3 \mathrm{~s}$ in the $\mathrm{T}^{+}$group (YC: $34.1 \pm 1.7 \mathrm{~s}$ ) and $35.2 \pm 1.1 \mathrm{~s}$ in the $\mathrm{T}^{-}$group (YC: $35.9 \pm 1.3 \mathrm{~s}$ ). Further examination of the first 10 sessions of training (5 blocks of 2 sessions) with repeated-measures ANOVA revealed an interaction of conditioning block (5) and group (4) $\left(F_{(12,128)}=2.774\right.$, $p=0.014)$. Namely, the ITI decreased across training in the $\mathrm{T}^{-}$ 
group from $40.1 \pm 2.4 \mathrm{~s}$ to $33.8 \pm 1.1 \mathrm{~s}$ (repeated-measures ANOVA, block: $\left.F_{(4,36)}=6.30, p=0.001\right)$ and in its respective YC group from $41.8 \pm 3.1 \mathrm{~s}$ to $33.8 \pm 1.5 \mathrm{~s}\left(F_{(4,24)}=7.37, p=0.001\right)$. In the $\mathrm{T}^{+}$and its respective YC groups, the ITI remained stable across conditioning $\left(F_{(4,40)}=0.082\right.$, not significant and $F_{(4,28)}=$ 0.48 , not significant, respectively). Group differences in ITI were present during the first two blocks of training (one-way ANOVAs: $F_{(3,35)}=4.75 / 3.73, p=0.008 / p=0.021$, respectively) after which the ITI in the $\mathrm{T}^{-}$and its respective $\mathrm{YC}$ group reached the level of the ITI in the $\mathrm{T}^{+}$and its respective $\mathrm{YC}$ group (oneway ANOVAs, blocks 3-5: $F_{(3,35)}=0.06-0.21$, not significant). To eliminate the effect of the differing ITI, learning and hippocampal responses during conditioning were compared between the $\mathrm{T}^{-}$and its respective $\mathrm{YC}$ groups and between the $\mathrm{T}^{+}$ and its respective $\mathrm{YC}$ groups. To further examine the effects of ITI on learning, we compared learning in the two YC groups: As a result of a longer ITI early in conditioning, a greater proportion of animals ( 4 of 7 vs 2 of 8 ) reached the $60 \%$ learning criterion $\left(\chi^{2}\right.$ test: $\left.\chi^{2}(1, N=7)=3.86, p=0.050\right)$. In addition, a longer ITI early in conditioning resulted in faster learning in animals that eventually learned (independent samples $t$ test on sessions to criterion: $\left.t_{(4)}=2.88, p=0.045\right)$. Reaching the $60 \%$ criterion took on average $14.5 \pm 1.5$ sessions in the $\mathrm{T}^{+} \mathrm{YC}$ group and $7.5 \pm 1.5$ sessions in the $\mathrm{T}^{-} \mathrm{YC}$ group.

During extinction (all sessions, 10), the ITI was on average $32.6 \pm 1.2 \mathrm{~s}$ in the $\mathrm{T}^{+}$group (YC: $32.0 \pm 0.8 \mathrm{~s}$ ) and $36.3 \pm 2.5 \mathrm{~s}$ in the $\mathrm{T}^{-}$group (YC: $33.3 \pm 1.8 \mathrm{~s}$ ). During extinction, no changes across training or differences between groups in the ITI (repeated-measures ANOVA, interaction: $F_{(12,60)}=0.77$, not significant, block: $F_{(4,60)}=1.56$, not significant, group: $F_{(3,15)}=$ 0.85 , not significant) occurred. Hence, the animals in the YC groups were combined into a single control group $(n=6$, average ITI $32.9 \pm 1.2 \mathrm{~s})$ and comparisons were made between the $\mathrm{T}^{-}$ $(n=7), \mathrm{T}^{+}(n=6)$ and the YC $(n=6)$ groups.

To study the effects of the consistency of the pretrial neural state between conditioning and extinction on extinction, the data were divided into two groups: Same $\left(\mathrm{T}^{+} / \mathrm{T}^{+}\right.$or $\left.\mathrm{T}^{-} / \mathrm{T}^{-}\right)$ and Different $\left(\mathrm{T}^{+} / \mathrm{T}^{-}\right.$or $\left.\mathrm{T}^{-} / \mathrm{T}^{+}\right)$. The average ITI was $34.9 \pm$ $1.4 \mathrm{~s}$ in the group Same $(n=7)$ and $34.2 \pm 3.0 \mathrm{~s}$ in the group Different $(n=6)$. Again, no changes across the extinction training or differences between the three groups (Same, Different, YC) in ITI (repeated-measures ANOVA, interaction: $F_{(8,64)}=0.45$, not significant, block: $F_{(4,64)}=1.55$, not significant, group: $F_{(2,16)}=0.28$, not significant) occurred. Together, possible differences between the groups $\left(\mathrm{T}^{+}\right.$vs $\mathrm{T}^{-}$vs YC and Same vs Different vs YC) in extinction could not be the result of differences in ITI.

\section{Training in the explicit absence of hippocampal $\theta$ leads to slower but better learning of trace eyeblink conditioning} The behavioral results of trace eyeblink conditioning and extinction are summarized in Figure 2. For conditioning, data were analyzed in two parts: $\mathrm{T}^{-}$versus its respective $\mathrm{YC}$ group and $\mathrm{T}^{+}$versus its respective YC group. This was done to avoid confounding effects of differences in the ITI (see ITI variation across training and between groups). Different animals had a different number of conditioning sessions (between 10 and 16) due to individual differences in learning speed. Hence, data from only the first 10 sessions (blocks 1-5) were subjected to repeated-measures ANOVA, and learning outcome was primarily assessed based on the number of animals that reached the learning criterion.

Conditioning led to increased blinking in response to the tone-CS in both the $\mathrm{T}^{+}$and the $\mathrm{T}^{+} \mathrm{YC}$ groups (repeated-measures
ANOVA, block: $F_{(4,68)}=3.75, p=0.024$; group: $F_{(1,17)}=0.19$, not significant; interaction: $F_{(4,68)}=0.80$, not significant) (for individual learning curves, see Fig. $2 A$ ). The same was true for the $\mathrm{T}^{-}$and the $\mathrm{T}^{-}$YC groups $\left(\right.$block: $F_{(4,60)}=13.53, p<0.001$; group: $F_{(1,15)}=$ 1.59 , not significant; interaction: $F_{(4,60)}=1.96$, not significant) (for individual learning curves, see Fig. 2B). Eventually, within a maximum of 16 conditioning sessions, 4 of 11 animals in the $\mathrm{T}^{+}$ group and 2 of 8 animals in the respective YC group reached the predetermined learning criterion of at least $60 \%$ conditioned responses within at least one conditioning session (Fig. 2C, left). The proportion of animals that learned in the $\mathrm{T}^{+}$group did not differ from that expected on the basis of the proportion of animals that learned in the respective YC group ( $\chi^{2}$ test: $\chi^{2}(1, N=$ $11)=0.76$, not significant). In the $\mathrm{T}^{-}$group, 9 of 10 animals learned the task compared with 4 of 7 in the respective $Y C$ group $\left(\chi^{2}\right.$ test: $\chi^{2}(1, N=10)=4.41, p=0.036$; see Fig. $2 C$, right $)$. To summarize, the proportion of animals that learned in the $\mathrm{T}^{-}$ group was significantly higher than the corresponding proportion in its respective YC group.

When the number of conditioning sessions needed to reach the $60 \%$ learning criterion was compared between the $\mathrm{T}^{+}$and the respective $\mathrm{YC}$ groups, no significant difference emerged (oneway ANOVA, $F_{(1,4)}=4.70$, not significant $/ p<0.1$ ) (see Fig. $2 D$, left). The same analysis for the $\mathrm{T}^{-}$and the respective $\mathrm{YC}$ groups indicated that animals that reached the learning criterion took significantly longer to do so in the $\mathrm{T}^{-}$group compared with the respective YC group (one-way ANOVA: $F_{(1,11)}=5.67, p=0.036$ ) (see Fig. 2D, right).

We also analyzed learning rate using the traditional $8 / 9$ criterion (see Materials and Methods). According to this criterion, $90 \%$ of animals in $\mathrm{T}^{-}$group learned, compared with $71 \%$ in the respective YC group ( $\chi^{2}$ test: $\chi^{2}(1, N=10)=1.69$, not significant). In the $\mathrm{T}^{+}$and corresponding $\mathrm{YC}$ groups, $46 \%$ compared with $63 \%$ of animals learned, respectively $\left(\chi^{2}\right.$ test: $\chi^{2}(1, N=$ $11)=3.21$, not significant). In terms of learning rate, the number of trials to criterion was on average $442 \pm 56$ in the $\mathrm{T}^{-}$group compared with $246 \pm 43$ in the corresponding YC group (oneway ANOVA: $\left.F_{(1,12)}=5.67, p=0.035\right)$. The mean number of trials to criterion in the $\mathrm{T}^{+}$group was $479 \pm 87$ compared with $539 \pm 70$ in the corresponding YC group (one-way ANOVA: $F_{(1,8)}=0.29$, not significant).

Further, we studied the number of trials needed to perform the fifth conditioned response. There was no difference in this measure between the $\mathrm{T}^{+}$and its $\mathrm{YC}$ group $(61 \pm 10$ vs $50 \pm 9$ trials, respectively, one-way ANOVA: $F_{(1,17)}=0.59$, not significant) or between the $\mathrm{T}^{-}$and its $\mathrm{YC}$ group $(54 \pm 10$ vs $45 \pm 9$ trials, $F_{(1,15)}=0.38$, not significant).

\section{Training contingent on $\boldsymbol{\theta}$ or contingent on a hippocampal state opposite to that used for conditioning, facilitated extinction}

Together, 19 animals learned trace eyeblink conditioning. These animals were further trained in extinction. For extinction, rabbits initially conditioned contingent upon either a high or low hippocampal $\theta$ ratio were redivided into groups so that half the animals remained in the same group (Same, $n=7$ ) and half were switched from the $\mathrm{T}^{+}$to the $\mathrm{T}^{-}$group or vice versa (Different, $n=6)$. Animals in the YC group remained in that group also for extinction $(n=6)$. Together, 6 animals were trained in extinction contingent on a high hippocampal $\theta$ ratio $\left(\mathrm{T}^{+}\right)$and 7 animals were trained contingent on a low hippocampal $\theta$ ratio $\left(\mathrm{T}^{-}\right)$. Contrary to conditioning, during extinction training, the ITIs were stable and comparable in all groups (see ITI variation across 

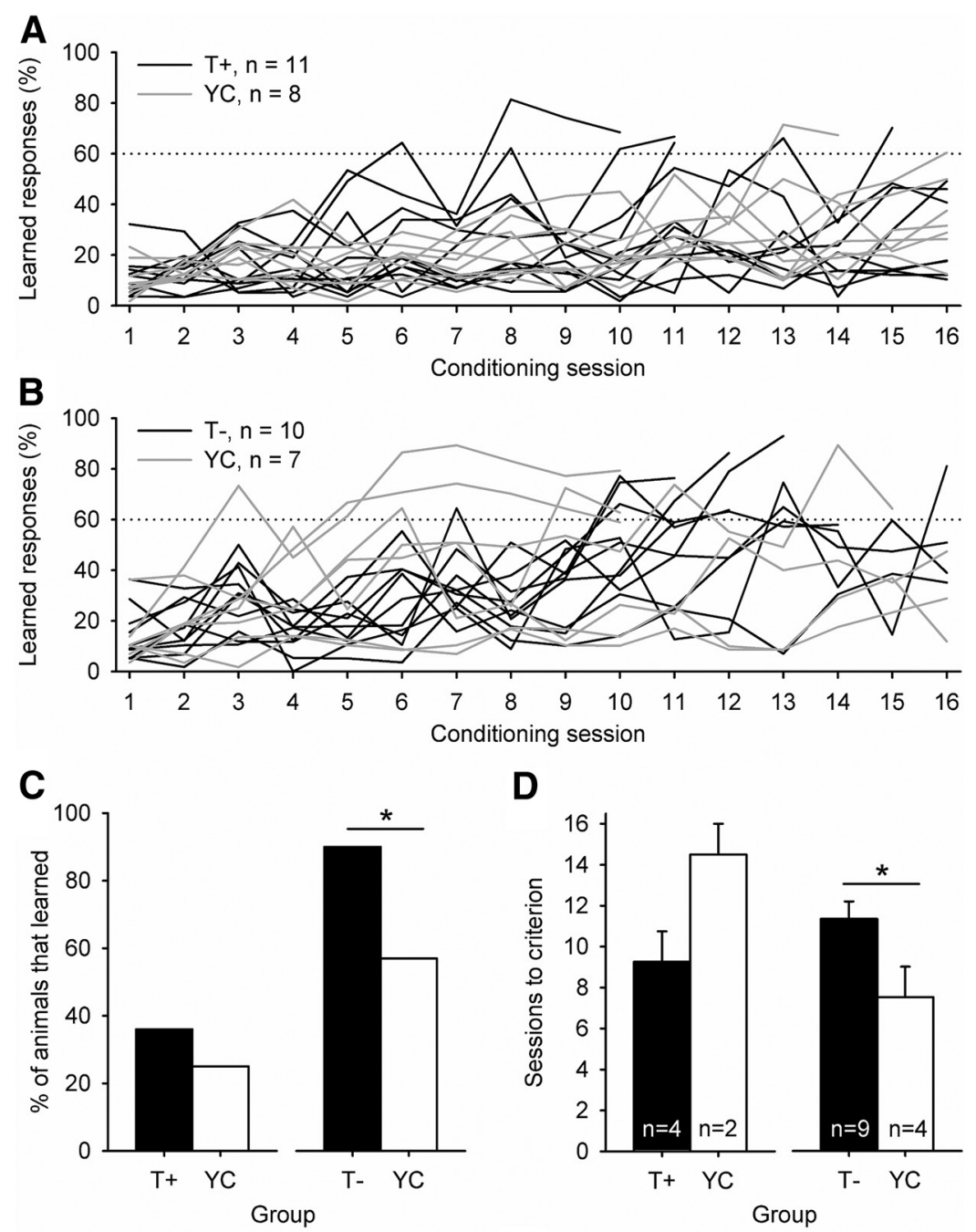

D

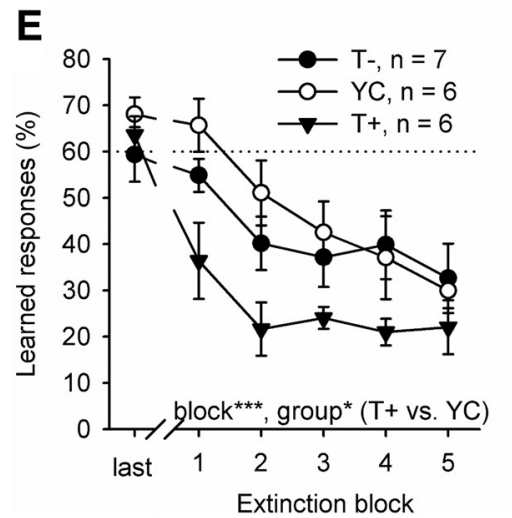

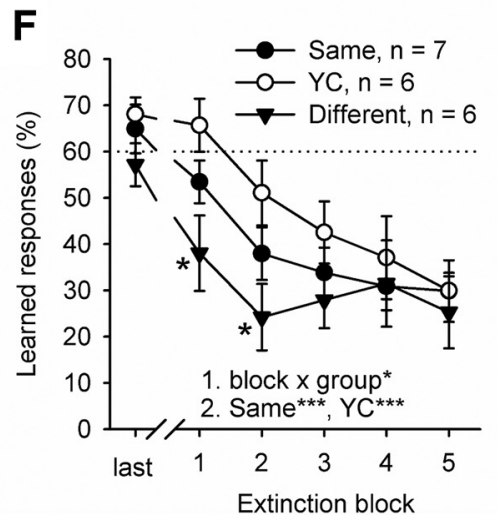

Figure 2. Training contingent on the explicit absence of hippocampal $\theta$ promoted trace eyeblink conditioning $(\boldsymbol{A}-\boldsymbol{D})$, whereas training contingent on dominant hippocampal $\theta$ facilitated extinction $(\boldsymbol{E})$. Further, extinction was facilitated when training was conducted in an internal state opposite to the one used to time training trials during the initial conditioning $(\boldsymbol{F})$. $\boldsymbol{A}, \boldsymbol{C}$, Animals trained in the presence of $\theta\left(\mathrm{T}^{+}\right)$and their corresponding $\mathrm{YC}$ group learned to a similar degree. $\boldsymbol{B}, \boldsymbol{C}$, Animals trained in the absence of $\theta\left(\mathrm{T}^{-}\right)$learned more often than animals in the corresponding $Y C$ group $\left(\chi^{2}, p<0.05\right)$. D , Animals that reached the learning criterion took significantly longer to do so in the $\mathrm{T}^{-}$group compared with the respective YC group (one-way ANOVA, $p<0.05$ ). $\boldsymbol{E}$, Compared with the YC group, extinction was facilitated in the group trained in the presence of dominant hippocampal $\theta\left(\mathrm{T}^{+}\right)$. $\boldsymbol{F}$, Compared with the YC group, extinction was also facilitated in animals trained in a neural state different to that used for conditioning (Different). The effect was limited to the first two blocks of extinction. $\boldsymbol{E}$, $\boldsymbol{F}$, Repeated-measures ANOVAs (aligned with the $x$-axis) and one-way ANOVAs (above or below data points): ${ }^{* *} p<0.001$; ${ }^{*} p<0.05$. Line number 1 indicates results of repeated-measures ANOVA with 3 groups and 5 blocks, and line number 2 indicates separate repeated-measures ANOVAs with 5 blocks for each group.

training and between groups); hence, the data from all the $\mathrm{YC}$ animals were pooled and comparisons then made between $\mathrm{T}^{+}$, $\mathrm{T}^{-}$, and $\mathrm{YC}$ groups and between the Same, Different, and YC groups.

First, we wanted to ensure that any differences in extinction were not the result of differences in the outcome of conditioning. One-way ANOVA conducted on the average CR percentage during the last block of conditioning indicated that the animals in the $\mathrm{T}^{-}, \mathrm{T}^{+}$, and $\mathrm{YC}$ groups that had reached the learning criterion had indeed learned to a comparable degree $\left(F_{(2,16)}=0.84\right.$, not significant) (Fig. $2 E)$. The same was true when animals in the $\mathrm{T}^{+}$and $\mathrm{T}^{-}$groups were grouped based on the consistency of the training paradigm during conditioning versus extinction: the animals in the Same, Different, and YC groups also showed similar levels of conditioned responding at the end of conditioning (one-way ANOVA, last block, $F_{(2,16)}=1.38$, not significant) (Fig. $2 F)$. Next, for the purpose of illustration and statistical testing, conditioned responding (\%) during extinction was averaged over two consecutive sessions to form 5 blocks.

Extinction led to decreased blinking in response to the tone-CS alone in the $\mathrm{T}^{+}$, the $\mathrm{T}^{-}$, and the $\mathrm{YC}$ group (repeatedmeasures ANOVA, block: $F_{(4,64)}=17.21$, $p<0.001$; interaction: $F_{(8,64)}=1.60$, not significant) (Fig. 2E). A significant effect of group was also observed: $F_{(2,16)}=4.06$, $p=0.037$. Post hoc comparisons between groups indicated that the $\mathrm{T}^{+}$group showed superior extinction compared with the YC group (Bonferroni-corrected $p=0.048)$. No difference emerged between either the $\mathrm{T}^{-}$and $\mathrm{T}^{+}$or between the $\mathrm{T}^{-}$and the $\mathrm{YC}$ group.

Next, we explored the effects of the consistency of the internal training context between conditioning and extinction training on learning extinction. Repeatedmeasures ANOVA revealed an interaction of group (Same vs Different vs YC) and extinction block $(5): F_{(8,64)}=2.26, p=$ 0.034 (Fig. $2 F$ ). Separate repeated-measures ANOVAs conducted for each group indicated that conditioned responding decreased as a function of extinction (5 blocks $)$ in the YC group $\left(F_{(4,20)}=14.83\right.$, $p<0.001)$ and in the group trained in the same state it had been conditioned in (group Same: $F_{(4,24)}=8.41, p<0.001$ ), whereas no change took place in the group conditioned in one state and trained in extinction in another state (group Different: $F_{(4,20)}=1.74$, not significant). Oneway ANOVAs further revealed that learned responding differed between the 

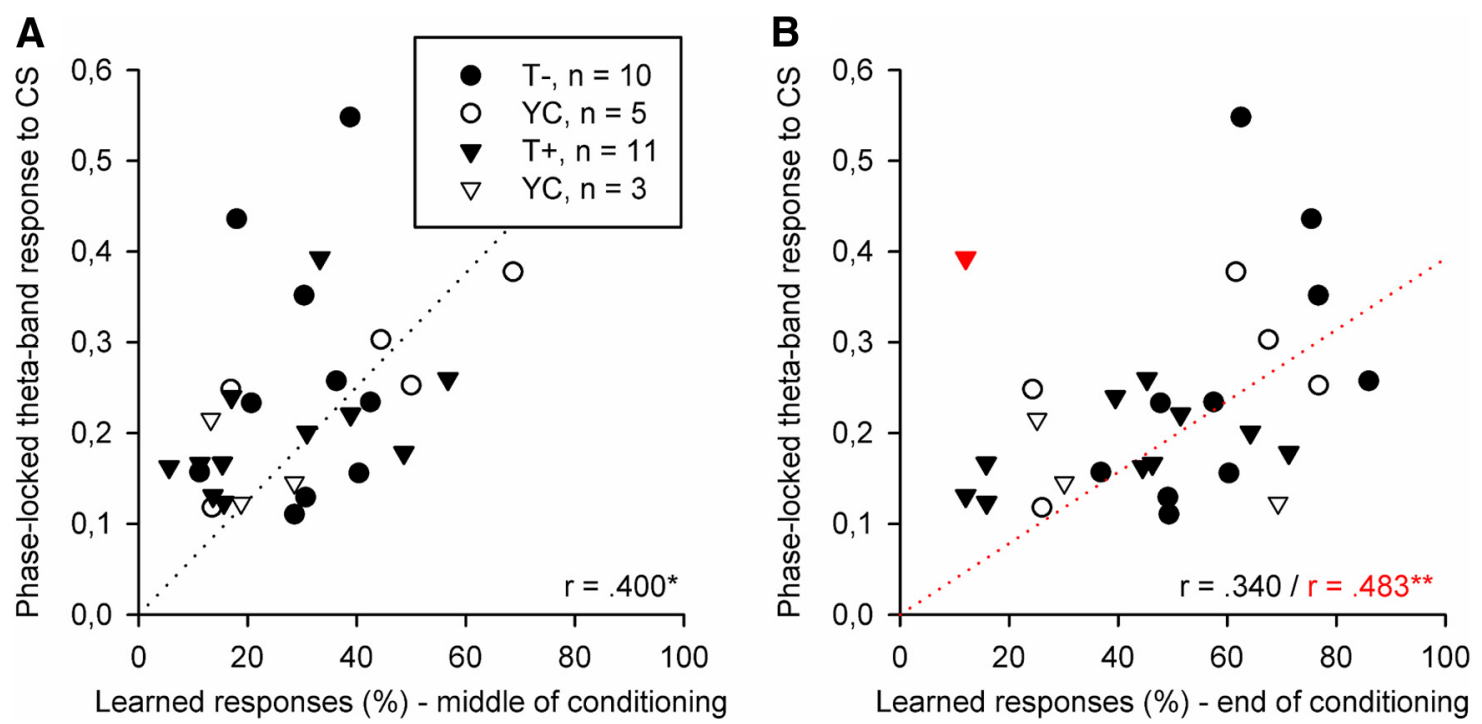

Figure 3. Hippocampal phase-locked $\theta$-band responses to the CS recorded early in training predicted learned responding in the middle $(\boldsymbol{A})$ and at the end $(\boldsymbol{B})$ of trace eyeblink classical conditioning. Pearson's correlation coefficients were calculated. ${ }^{* *} p<0.01 .{ }^{*} p<0.05$. Dotted lines indicate linear regression. $\boldsymbol{B}$, Results are presented including (black) and excluding (red) the outlier marked with a red triangle.

groups during the first $\left(F_{(2,16)}=4.74, p=0.024\right)$ and second $\left(F_{(2,16)}=3.94, p=0.041\right)$ blocks of extinction. Specifically, conditioned responding was lower in the Different group compared with the YC group during the first two blocks of extinction ( $p=$ $0.022 / p=0.038)$. In other words, animals that had been switched from $\mathrm{T}^{+}$to $\mathrm{T}^{-}$or from $\mathrm{T}^{-}$to $\mathrm{T}^{+}$training between conditioning and extinction very rapidly ceased responding to the tone-CS.

Phase-locked hippocampal $\theta$-band $(4-8 \mathrm{~Hz})$ responses to the CS predicted learning during trace eyeblink conditioning

Phase-locking of hippocampal $\theta$-band responses to the CS measured using the PLV (see Materials and Methods) was compared between groups and across training $\left(\mathrm{T}^{+}\right.$vs its $\mathrm{YC}$ group and $\mathrm{T}^{-}$ vs its YC group for conditioning and $\mathrm{T}^{+}$vs $\mathrm{T}^{-}$vs YC for extinction). Repeated-measures ANOVAs did not reveal any significant differences between groups or changes across training during either conditioning or extinction (data and exact statistics not shown). Hence, data from all groups were pooled to facilitate more straightforward study of the connection between phaselocked $\theta$-band responses and learning. However, repeatedmeasures ANOVAs did not reveal any significant differences between groups or changes across training when phase-locked $\theta$-band responses to the CS elicited during conditioning were compared between animals that learned $(n=17)$ versus animals that did not learn $(n=12)$ (data and exact statistics not shown).

Next, correlations were calculated between hippocampal responses to the CS and learning. Well phase-locked hippocampal $\theta$-band responses to the CS early on during trace eyeblink conditioning (first block) predicted good learning measured as the percentage of behavioral learned responses elicited (blocks 3-5, $r=0.396-0.429, p=0.020-0.034, n=29$ ). To better quantify learning in the middle of conditioning, the average CR percentage during the two middle sessions (rounding down, if needed) was calculated for each animal. This measure correlated significantly with the $\theta$-band PLV to the CS recorded during the first conditioning block: $r=0.400, p=0.032$ (Fig. 3A). The phaselocking of hippocampal $\theta$-band responses to the CS during the first conditioning block also predicted learned responding at the end of conditioning (Fig. $3 B$ ), although the connection was weaker: $r=0.340$, not significant, $p<0.1$. As one animal (in the $\mathrm{T}^{+}$group) had very well phase-locked $\theta$-band responses yet did not learn, it was classified as an outlier. When removed from the data, the correlation between hippocampal responses and learning at the end of conditioning also became significant: $r=0.483, p=0.009, n=28$. No significant correlations were found between the phase-locked hippocampal $\theta$-band responses to the CS and measures of learning rate (session to $60 \%$ CRs/TTC/trials to fifth CR: $n=17 / 20 / 29, r=-0.34 /-20 / 27$, not significant).

We also calculated the correlations between the phase-locked hippocampal $\theta$-band responses to the CS early in the extinction training (first block) and conditioned responding throughout extinction ( 5 blocks) and found no significant correlations $(n=$ $16, r=0.044-0.254$, not significant).

\section{Discussion}

We set out to study how training contingent on a state characterized by the presence versus absence of hippocampal $\theta$ activity affects trace eyeblink conditioning and subsequent extinction. Contrary to our hypothesis and previous results (Griffin et al., 2004), training contingent on a high $\left(\mathrm{T}^{+}\right)$level of hippocampal $\theta$ did not affect trace eyeblink conditioning. Curiously, learning was more probable, yet required more trials, in animals that were trained in the absence of hippocampal $\theta\left(\mathrm{T}^{-}\right)$compared with their yoked controls. As hypothesized, training contingent on high $\theta$ promoted extinction. In addition, like training in one external environment and testing in another results in a decrease in memory retrieval (Godden and Baddeley, 1975), changing the hippocampal state in which the subject was conditioned versus trained in extinction facilitated extinction. Finally, we examined whether the pretrial oscillatory state determines the subsequent hippocampal response to the CS and thus predicts learning. Contrary to our hypothesis, responses did not differ based on the pretrial hippocampal oscillatory state. However, as hypothesized, hippocampal $\theta$-band responses reliably predicted learning during conditioning (but not extinction).

We found that, although learning is slower, trace eyeblink conditioning leads to a robust learned response more often if 
trials are presented in the absence of hippocampal $\theta\left(\mathrm{T}^{-}\right)$. This is in line with previous findings of improved learning when conditioning trials are presented contingent on hippocampal ripples (Nokia et al., 2010) and of slower learning in the absence of $\theta$ (Griffin et al., 2004). However, training contingent on the absence of $\theta$ enhanced learning only after prolonged training, whereas ripple-contingent training facilitated the commencement of conditioned responding early in training (Nokia et al., 2010). The state in which ripples occur is clearly more uniform than the non $\theta$ state, a difference that might explain the difference in the timing of the effects. Contrary to our hypothesis, as well as earlier findings on the connection between $\theta$ and learning (Seager et al., 2002; Griffin et al., 2004), presenting conditioning trials contingent on dominant hippocampal $\theta\left(\mathrm{T}^{+}\right)$had no effect on learning outcome in our present experiment. There was a trend $(p<0.1)$ for animals in the $\mathrm{T}^{+}$group that did learn to do so faster than their YCs, which would fit with previous findings by Griffin et al. (2004). The discrepancy between our results and those in the aforementioned study could be related to methodological differences, such as the definition of the $\mathrm{T}^{+}$and $\mathrm{T}^{-}$states, the ITI, or the parameters of the stimuli used for conditioning. It might be, for example, that $\mathrm{T}^{+}$conditioning only affects learning outcome if the CS and/or US is presented to the trough of the fissure $\theta$, when input from the entorhinal cortex dominates and longterm potentiation occurs at the CA3-CA1 synapses (Hasselmo et al., 2002). In our experiment, the phase of $\theta$ was random at both CS and US onset. Further, the data and analyses presented by Griffin et al. (2004) concentrated on early acquisition, whereas we trained animals up to $16 \mathrm{~d}$ and only found a facilitative effect of $\mathrm{T}^{-}$treatment on conditioning very late in training. Thus, it is difficult to make straightforward comparisons between the two studies. Nevertheless, if the transient state in which $\theta$-band activity dominates hippocampal electrophysiological oscillations generally promotes learning, the $\mathrm{T}^{+}$group in our study should have learned trace eyeblink conditioning significantly better and/or faster.

In our study, $\mathrm{T}^{+}$training facilitated extinction, whereas $\mathrm{T}^{-}$ training had no effect on it. This is in line with our previous finding of slower extinction when the CS is presented contingent on hippocampal ripples (Nokia et al., 2010). These results suggest that pretrial neural states and resultant behavior during extinction training constitute a continuum, with dominant $\theta$ and inhibition of responding at one end, no- $\theta$ somewhere in the middle, and ripples and heightened responding at the other extreme. This seems plausible because the state in which ripples occur promotes excitatory responding in the hippocampus (Buzsáki, 1986), whereas CA1 pyramidal cells rarely fire action potentials during periods of dominant $\theta$ (Kamondi et al., 1998). Instead of faster learning of the new "CS - no-US" association, the expedited extinction in the $\mathrm{T}^{+}$group could be the result of a failure in memory retrieval and/or the performance of the conditioned response. Interestingly, in the present experiment, extinction was also facilitated in animals that were initially conditioned contingent on one state and later extinguished contingent on a different state. This is in line with our hypothesis suggesting that the internal neural state can act as a context for learning and memory similar to the external environment: retrieval is known to be deteriorated when memory is tested in an environment different from that where it was acquired (Godden and Baddeley, 1975; Gordon et al., 1981). Of course, state-inconsistent extinction could also just expedite learning that the CS is no longer followed by an US. Before any further speculation about these results, the dilemma between expedited learning and a simple retrieval/per- formance deficit should be addressed in future studies by incorporating a relearning or testing phase after extinction training.

In addition to behavior, we also analyzed phase-locked hippocampal $\theta$-band $(4-8 \mathrm{~Hz})$ responses to the CS. The pretrial hippocampal state did not have an effect on the responses either during conditioning or during extinction. This is at odds with our hypothesis and the behavioral results reviewed above. It seems that regulating the pretrial hippocampal state to control learning affected information processing in the hippocampus in a way not detectable with our methods and/or in circuits crucial for learning outside the hippocampus, perhaps, for example, in the cerebellum or in the medial prefrontal cortex (Weiss and Disterhoft, 2011). However, regardless of experimental group, phase-locked hippocampal $\theta$-band responses to the CS predicted learning: the better phase-locked the responses early in training, the more conditioned responses the animal elicited in the middle and end of conditioning. This observation is in line with our hypothesis and with previous results (Nokia et al., 2009, 2010) indicating higheramplitude and better phase-locking of hippocampal $\theta$-band responses to the CS early in training in animals that eventually learn trace eyeblink conditioning. The result is not surprising because it was suggested as early as during the 1970s that the phaselocking of hippocampal $\theta$ activity in response to a CS accompanies learning its significance (Buzsáki et al., 1979). It could be that events evoking a highly temporally uniform response in the hippocampus from one occasion to another are processed more accurately and efficiently (perhaps throughout the brain) and that this leads to efficient learning. Via oscillatory synchronization (Fries, 2005), temporally regular responses to repeated stimuli could enable (at least in theory) efficient signaling between the brain structures involved in learning about those stimuli (Hoffmann and Berry, 2009; Wikgren et al., 2010) and hence promote plasticity (Hebb, 1949). At the psychological level, our interpretation of hippocampal phase-locked $\theta$-band responses to external stimuli is that they reflect the level of selective attention paid to that specific stimulus: highly phase-locked responses reflect strong attention, whereas lack of regularly repeating responses indicates low attention (see also Vinogradova et al., 1993).

In conclusion, current and previous findings on hippocampal state-contingent conditioning and extinction suggest that the notion of ongoing, spontaneously occurring hippocampal $\theta$ oscillations as an all-encompassing promoter of learning is too simplistic. Although an overall high level of hippocampal $\theta$, possibly indicating a predisposition of an individual to be aroused and attentive toward its surroundings rather than mellow, seems to consistently predict good learning (Berry and Thompson, 1978; Nokia et al., 2009; Nokia et al., 2012a), effects of $\theta$-triggered training depend on the task at hand and are more variable. We suggest that any distinguishable spontaneously generated (hippocampal) oscillatory state can affect learning by setting the stage for excitatory versus inhibitory responding at the neural and behavioral levels, and perhaps also by serving as an additional cue or context for learning, much like the features of the external environment, at the psychological level. It would seem that states that occur relatively rarely, such as the explicit absence of $\theta$ oscillations, might serve as more distinct cues and thus affect learning more than states that take place relatively often, such as the high $\theta$ state. However, rather than the preevent activity, in the context of acquiring a conditioned response, learning is predicted by the temporal regularity of hippocampal responses to the event early in training. We suggest that, at the psychological level, the temporally regular responses translate onto selective attention. 


\section{References}

Berry SD, Thompson RF (1978) Prediction of learning rate from the hippocampal electroencephalogram. Science 200:1298-1300. CrossRef Medline

Buzsáki G (1986) Hippocampal sharp waves: their origin and significance. Brain Res 398:242-252. CrossRef Medline

Buzsáki G (1989) Two-stage model of memory trace formation: a role for "noisy" brain states. Neuroscience 31:551-570. CrossRef Medline

Buzsáki G, Moser EI (2013) Memory, navigation and $\theta$ rhythm in the hippocampal-entorhinal system. Nat Neurosci 16:130-138. CrossRef Medline

Buzsáki G, Grastyán E, Tveritskaya IN, Czopf J (1979) Hippocampal evoked potentials and EEG changes during classical conditioning in the rat. Electroencephalogr Clin Neurophysiol 47:64-74. CrossRef Medline

Chrobak JJ, Buzsáki G (1996) High-frequency oscillations in the output networks of the hippocampal-entorhinal axis of the freely behaving rat. J Neurosci 16:3056-3066. Medline

Fries P (2005) A mechanism for cognitive dynamics: neuronal communication through neuronal coherence. Trends Cogn Sci 9:474-480. CrossRef Medline

Godden DR, Baddeley AD (1975) Context-dependent memory in two natural environments: on land and under water. $\mathrm{Br}$ J Psychol 66:325-331. CrossRef

Gordon WC, McCracken KM, Dess-Beech N, Mowrer RR (1981) Mechanisms for the cueing phenomenon: the addition of the cueing context to the training memory. Learn Motiv 12:196-211. CrossRef

Green JD, Arduini AA (1954) Hippocampal electrical activity in arousal. J Neurophysiol 17:533-557. Medline

Griffin AL, Asaka Y, Darling RD, Berry SD (2004) $\theta$-contingent trial presentation accelerates learning rate and enhances hippocampal plasticity during trace eyeblink conditioning. Behav Neurosci 118:403-411. CrossRef Medline

Hasselmo ME, Bodelón C, Wyble BP (2002) A proposed function for hippocampal $\theta$ rhythm: separate phases of encoding and retrieval enhance reversal of prior learning. Neural Comput 14:793-817. CrossRef Medline

Hebb DO (1949) The organization of behavior. New York: Wiley.

Hoffmann LC, Berry SD (2009) Cerebellar $\theta$ oscillations are synchronized during hippocampal $\theta$-contingent trace conditioning. Proc Natl Acad Sci U S A 106:21371-21376. CrossRef Medline

Kamondi A, Acsády L, Wang XJ, Buzsáki G (1998) $\theta$ oscillations in somata and dendrites of hippocampal pyramidal cells in vivo: activitydependent phase-precession of action potentials. Hippocampus 8:244-261. CrossRef Medline

Nokia MS, Wikgren J (2010) Hippocampal $\theta$ activity is selectively associated with contingency detection but not discrimination in rabbit discrimination-reversal eyeblink conditioning. Hippocampus 20:457460. CrossRef Medline

Nokia MS, Penttonen M, Korhonen T, Wikgren J (2009) Hippocampal $\theta$-band activity and trace eyeblink conditioning in rabbits. Behav Neurosci 123:631-640. CrossRef Medline

Nokia MS, Penttonen M, Wikgren J (2010) Hippocampal ripple-contingent training accelerates trace eyeblink conditioning and retards extinction in rabbits. J Neurosci 30:11486-11492. CrossRef Medline

Nokia MS, Sisti HM, Choksi MR, Shors TJ (2012a) Learning to learn: $\theta$ oscillations predict new learning, which enhances related learning and neurogenesis. PLoS One 7:e31375. CrossRef Medline

Nokia MS, Mikkonen JE, Penttonen M, Wikgren J (2012b) Disrupting neural activity related to awake-state sharp wave-ripple complexes prevents hippocampal learning. Front Behav Neurosci 6:84. CrossRef Medline

Palva JM, Palva S, Kaila K (2005) Phase synchrony among neuronal oscillations in the human cortex. J Neurosci 25:3962-3972. CrossRef Medline

Seager MA, Johnson LD, Chabot ES, Asaka Y, Berry SD (2002) Oscillatory brain states and learning: impact of hippocampal $\theta$-contingent training. Proc Natl Acad Sci U S A 99:1616-1620. CrossRef Medline

Vinogradova OS, Brazhnik ES, Kitchigina VF, Stafekhina VS (1993) Acetylcholine, $\theta$-rhythm and activity of hippocampal neurons in the rabbit: IV. Sensory stimulation. Neuroscience 53:993-1007. CrossRef Medline

Weiss C, Disterhoft JF (2011) Exploring prefrontal cortical memory mechanisms with eyeblink conditioning. Behav Neurosci 125:318-326. CrossRef Medline

Wikgren J, Nokia MS, Penttonen M (2010) Hippocampo-cerebellar $\theta$ band phase synchrony in rabbits. Neuroscience 165:1538-1545. CrossRef Medline 